\title{
DISPOSITIVO PARA COLETA E PROCESSAMENTO DE SINAIS CEREBRAIS E SUA INTEGRAÇÃO A CADEIRA DE RODAS
}

\author{
Carlos Alberto Nascimento ${ }^{1}$, Prícilla Karen Suzano ${ }^{1}$, Lucas Custódio ${ }^{1}$, Diana F. \\ Adamatti $^{1}$ \\ ${ }^{1}$ Centro de Ciências Computacionais - Universidade Federal do Rio Grande (FURG) \\ Av. Itália Km 8, 96201-900 Rio Grande, Rio Grande do Sul \\ carlos.longo@yahoo.com.br, prikarensuzano@gmail.com, \\ lucascustodio@hotmail.com.br, dianaadamatti@furg.br
}

\begin{abstract}
In order to facilitate and guarantee access to mobility for citizens who have quadriplegia, this article introduces the concept of an electric wheelchair controlled by neural impulses for people who cannot use other movement control interfaces. This process is done by creating a device to collect brain stimuli by performing recognition and filtering of these signals, in order to generate directional commands for a wheelchair.

Resumo. Visando facilitar e garantir o acesso a mobilidade de cidadãos que possuem tetraplegia, esse artigo apresenta o conceito de uma cadeira de rodas elétrica controlada pelos impulsos neurais para pessoas que não podem usar outras interfaces de comando de movimentos. Esse processo é feito através da criação de um dispositivo para coleta de estímulos cerebrais realizando reconhecimento e filtragem desses sinais, de modo a gerar comandos direcionais para uma cadeira de rodas.
\end{abstract}

\section{Introdução}

Indivíduos com deficiências neuromusculares, em especial as causadas por lesão medular, possuem uma condição de insuficiência parcial ou total do funcionamento da medula espinhal decorrente da interrupção dos tratos nervosos motor e sensorial deste órgão. Essa condição pode levar a alterações nas funções motoras e déficits sensitivos, superficial e profundo nos segmentos corporais localizados abaixo do nível da lesão. (Cerezetti, 2012). Isso se mostra uma relevante problemática, pois segundo a organização mundial da saúde (WHO - World Health Organization), a cada ano, ao redor do mundo, entre 250.000 e 500.000 pessoas sofrem de lesão da medula espinhal.

Os casos de lesão na medula podem atingir diferentes níveis de gravidade, sendo a tetraplegia ou quadriplegia o ápice, que é quando a paralisia afeta todas as quatro extremidades do corpo do indivíduo, juntamente à musculatura do tronco. Essa é, frequentemente, uma consequência irreversível. O tetraplégico é uma pessoa que tem necessidade de assistência contínua durante toda a sua vida para realizar quaisquer ações necessárias do cotidiano, como por exemplo higiene básica e locomoção.

Visando atender esses cidadãos, se faz necessário o acesso a dispositivos de assistência apropriados que podem permitir que as pessoas realizem atividades diárias que de outra forma não seriam capazes de realizar, reduzindo as limitações funcionais e a dependência (WHO, 2013). Dessa forma, é proposto o desenvolvimento de um meio diferente de identificação de comandos locomotores, que venha a dar a possibilidade desse indivíduo de se deslocar para os locais desejados sem a necessidade de assistência. 
Esse projeto faz o uso de um dispositivo de BCI (Brain-Computer Interface). BCI, ou interface cérebro-computador, é a tecnologia que traz uma opção de comunicação para pessoas com deficiências neuromusculares que são impedidas de exercer atividades convencionais de comunicação aumentativa. BCI fornece a esses usuários canais de comunicação que não dependem dos nervos e músculos periféricos (Wolpaw et al., 2000). É um sistema de comunicação que permite ao usuário enviar comandos para um computador por meio de variações de atividade cerebral, que por sua vez são medidas e processadas pelo sistema (Renard et al., 2010).

\section{Fundamentação Teórica}

\subsection{Funcionamento do Cérebro}

O cérebro humano é o órgão mais complexo do corpo humano, sendo responsável por processar informações, realizar memorização, regular as funções dos órgãos entre outros. Ele é dividido em quatro regiões, com cada uma delas tendo uma função específica. Entre algumas atividades que ele comanda, temos as de controle das ações motoras, a integração dos estímulos sensoriais e as atividades neurológicas como a memória e a fala. As regiões do cérebro e seus papéis são:

- Lobo Frontal: É o maior dos quatro. Responsável pelos mais simples movimentos físicos, funções de aprendizado, pensamento, memória funcional e a linguagem.

- Sulco Central: É responsável pela motricidade e sensibilidade. É ele que separa o lobo parietal do lobo frontal.

- Lobo Temporal: É responsável por perceber e reconhecer os estímulos auditivos e os vinculados à memória e à emoção.

- Lobo Parietal: É responsável pela percepção espacial e informações sensoriais de tato, dor, calor e frio. Também intervém na sensação de equilíbrio.

- Lobo Occipital: É responsável por captar e processar os dados visuais, é aqui que se encontra o córtex visual.
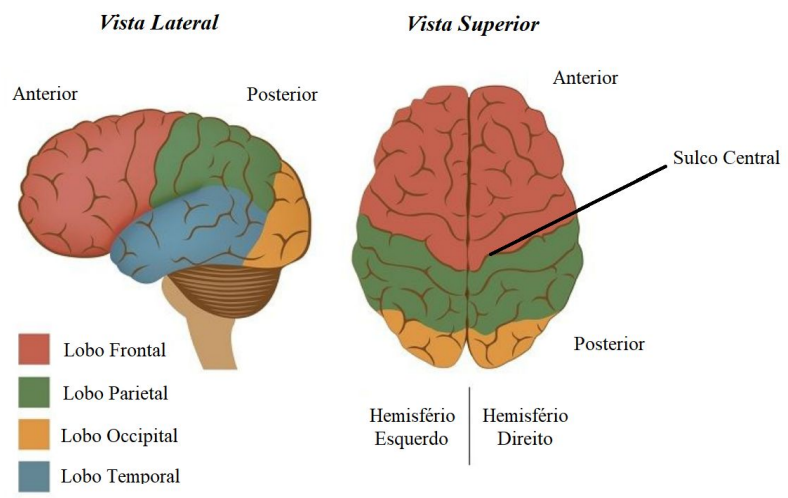

Figura 1. Identificação dos lobos cerebrais (Toda Matéria, 2020).

O cérebro gera ondas cerebrais diferentes de acordo com o tipo de atividade que está sendo realizada pelo indivíduo. Podemos separar essas ondas cerebrais por diferentes intervalos de frequências (Sanei e Chambers, 2007). São elas:

- Alpha $(\alpha)$ : Geradas em um estado de calma, geralmente quando o indivíduo está meditando ou até mesmo descansando mas sem dormir. Variam de 8 a $13 \mathrm{~Hz}$. 
- Theta $(\theta)$ : Relacionada a capacidades imaginativas e com emoções muito profundas. Variam de 4 a $7 \mathrm{~Hz}$.

- Beta $(\beta)$ : Geradas em um momento de atividade neuronal intensa como dirigir, fazer uma prova e escrever a monografia. Variam de 13 a $30 \mathrm{~Hz}$.

- Delta $(\delta)$ : Ondas com uma alta amplitude, geralmente relacionada a sono profundo. Variam de 0.5 a $4 \mathrm{~Hz}$.

- Gamma $(\gamma)$; Surge com tarefas de alto processamento cognitivo. Maior que 35 $\mathrm{Hz}$.

\subsection{Estímulos Visuais}

A identificação e processamento dos sinais cerebrais é realizada através do método Steady State Visually Evoked Potential (SSVEP), ou potenciais visualmente evocados no estado de equilíbrio (Iscan \& Nikulin, 2018). São sinais gerados por estímulos visuais luminosos que oscilam em determinadas frequências constantes. Quando a retina recebe um estímulo visual luminoso que varia de $3,5 \mathrm{~Hz}$ a $75 \mathrm{~Hz}$, o cérebro tende a gerar atividade elétrica com a mesma frequência ou em frequências múltiplas as do estímulo. A Figura 2 ilustra a asserção onde serão utilizados os eletrodos nas $\mathrm{O} 1, \mathrm{O} 2, \mathrm{Oz}$, ilustrados na tonalidade azul e P3, P4, P7, P8, P9, P10 e Pz, ilustrados na tonalidade verde.

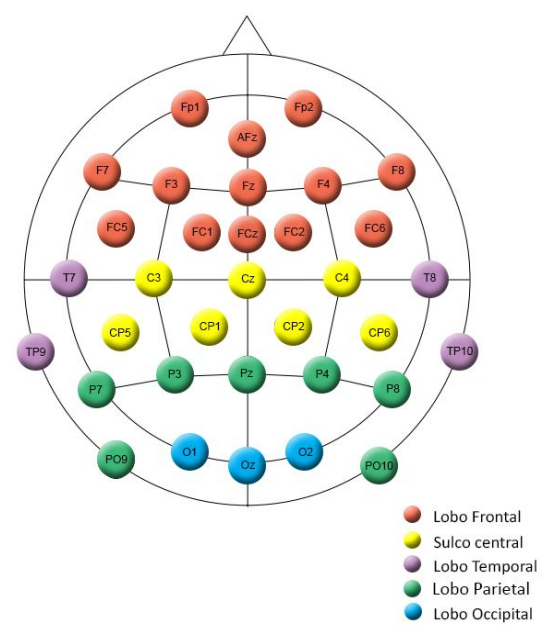

\section{Figura 2. Regiões que cada grupo de eletrodo captura (Bastos, Adamatti \& Carvalho, 2015).}

\section{Métodos e Materiais}

Para a coleta da atividade elétrica cerebral será feito o uso do amplificador ActiCHamp de eletroencefalograma (EEG), posicionado no couro cabeludo no qual 10 eletrodos são inseridos de modo não invasivo para fazer o contato com o cérebro do indivíduo. O actiCHamp (amplificador de canal ativo) é um amplificador de 24 bits que pode ser usado com 32, 64, 96, 128 e 160 canais EEG. (ActiCHamp, 2019).

A leitura dos dados coletados é realizada pelo software OpenViBE, usado para aplicações de neurociências em tempo real, isto é, para processamento em tempo real de sinais cerebrais. (OpenVibe, 2018). O processamento desses sinais é feito utilizando a biblioteca MNE, software Python de código aberto para explorar, visualizar e analisar dados neurofisiológicos humanos (MNE, 2019). Tal biblioteca foi escolhida a fim de 
reduzir a quantidade de processamento utilizada pois para a qual, o OpenViBE demandava muito mais do hardware.

A comunicação entre o MNE e o OpenViBE é feita utilizando o protocolo de comunicação Lab Streaming Layer (LSL, 2019) que serve como um canal de comunicação em tempo real. Para este projeto, foi desenvolvida uma aplicação na linguagem de programação Python, com o objetivo de enviar dados direcionais através de conexão bluetooth a uma placa Arduino UNO, responsável por realizar a movimentação de um protótipo de cadeira de rodas.

Foi escolhido o sistema operacional Windows 10 para execução do software. Apesar de ser um sistema operacional que demanda de mais processamento, memória e espaço interno que o Ubuntu, tal escolha foi necessária devido a uma série de incompatibilidades do Ubuntu com o OpenViBE. O computador utilizado para hospedar o sistema operacional é um notebook com processador i5 $8250 \mathrm{U}$ e $8 \mathrm{~GB}$ de memória RAM.

Em conjunto com o OpenViBE é executado a aplicação desenvolvida em Python responsável por toda a identificação, filtragem, processamento das ondas cerebrais e conexão do hardware que está sendo utilizado. A Figura 3 apresenta a esquemática dos componentes utilizados nesse projeto.

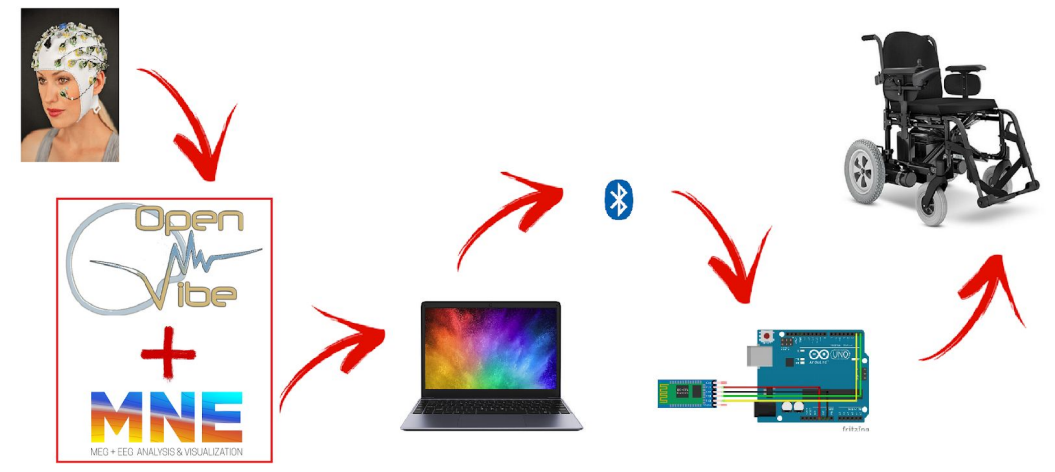

Figura 3. Esquemático do hardware e software utilizados.

\subsection{Aplicação Utilizando SSVEP}

O sistema BCI-SSVEP recebe os estímulos visuais, que são reproduções de caixas luminosas em frequências oscilantes específicas, estes estímulos são identificados por causarem picos de sinal cerebral. Cada estímulo provoca uma reação no cérebro em uma frequência que controla determinada aplicação BCI, tais como, andar para frente e para trás, andar rotacionando para a direita, andar rotacionando para a esquerda e por fim, parar (quaisquer frequências diferentes das anteriores).

O usuário apenas deve focalizar a atenção para o estímulo correspondente a ação desejada para que o sistema realize o movimento na cadeira de rodas.

\subsection{Protótipo da Cadeira de Rodas}

Para fins de teste, o modelo da cadeira de rodas utilizado foi montado com uma plataforma motorizada para Arduino. O kit é composto por um Arduino UNO, dois motores DC que realizam a movimentação das rodas, uma ponte H L298N responsável pela distribuição de energia para os motores e um módulo bluetooth (Módulo Bluetooth 
RS232 HC-05) que permite a comunicação entre o sistema e o Arduino. Todo o esquema é alimentado por duas baterias de $9 \mathrm{~V}$.

\section{Conclusão}

Já foram realizados testes para verificar a efetividade do método SSVEP para a identificação e processamento dos sinais cerebrais, os testes foram realizados com datasets colhidos pela equipe de desenvolvimento. Os resultados obtidos apontam que o método é eficaz para a identificação dos estímulos separados por frequências, possibilitando a verificação de qual movimento o indivíduo deseja realizar na cadeira de rodas.

Temos como próximos passos a filtragem dos dados adquiridos pela toca, pois o processo de aquisição dos dados captados pelos eletrodos é contaminado com ruído que pode vir de diferentes fontes, como piscar os olhos ou pensar em movimentar um membro. Portanto, é preciso realizar um pré-processamento para eliminar esses ruídos chamados artefatos e extrair um sinal livre desses ruídos, conservando por fim dados confiáveis para melhor extrairmos as informações desejadas.

O processo de filtragem deve ocorrer para cada epoch que é recebido de cada eletrodo. Epochs são dados agrupados em um intervalo de tempo. O primeiro processo é retirar somente a frequência desses epochs.

Utilizando dois filtros iniciais de banda alta e baixa para separar somente as frequências entre $3.5 \mathrm{~Hz}$ e $75 \mathrm{~Hz}$ para serem analisadas. Após isso, é feita a média de cada onda captada, deixando-as com um aspecto menos ruidoso.

Apesar da leitura dos sinais cerebrais ser realizada de maneira precisa, não há homogeneidade no valor das frequências, pois os eletrodos estão distribuídos em torno de uma área. Tomando como exemplo o valor alvo de frequência $13 \mathrm{~Hz}$, sabemos que iremos encontrar valores com relativa discrepância, variando para mais ou para menos em torno desse valor alvo. Portanto, de forma a uniformizar esses valores, devemos utilizar métodos de classificação das frequências nessas amostras captadas. De modo a realizar essas técnicas, iremos utilizar modelos regressivos de machine learning.

Serão utilizados dois métodos, o CSP (Common spatial pattern ou padrão espacial comum) para extrair as características e o LDA (Linear Discriminant Analysis ou Análise Discriminante Linear) para a classificação.

Um limitador dos testes e resultados do trabalho apresentado é o hardware do protótipo da cadeira de rodas, por esse motivo pretende-se substituir o protótipo atual por uma cadeira de rodas motorizada. $\mathrm{O}$ objetivo é produzir testes mais realísticos do uso no dia a dia e dessa forma obter mais resultados embasando a efetividade do dispositivo proposto nesse artigo. No entanto, esses testes são limitados à áreas de maior segurança e controle para não gerar quaisquer danos ao indivíduo que estiver o testando.

\section{Referências}

ActiCHamp (2019). Brain Vision LLC: Solution for neuropsychological research. https://brainvision.com/actichamp. html. Acesso em: 2 de março 2020.

Bastos, N., Carvalho, F. A. H., \& Adamatti, D. F.. (2015) Ensino de Lógica de Programação no Ensino Médio e suas implicações na Neurociências. In: XXVI 
Simpósio Brasileiro de Informática na Educação, 2015, Maceió / AL. SBIE 2015. Porto Alegre / RS: SBC, 2015. p. 1-10.

Cerezetti, C. R. N., Nunes, G. R., Cordeiro, D. R. C. L., \& Tedesco, S. A.. (2012) Lesão Medular Traumática e estratégias de enfrentamento: revisão crítica. São Paulo. http://bvsms.saude.gov.br/bvs/artigos/mundo_saude/lesao_medular_traumatica_estra tegias_enfrentamento.pdf

Iscan, Z., \& Nikulin, V. V.. (2018). Steady state visual evoked potential (SSVEP) based brain computer interface (BCI) performance under different perturbations. PLoS One,13(1).

LSL (2019). Sistema para coleta unificada de medidas de séries temporais. https://github.com/sccn/labstreaminglayer. Acesso em: 2 de março 2020.

MNE (2019). Software de código aberto para explorar, visualizar e analisar dados neurofisiológicos humanos. https://mne.tools/stable/index.html. Acesso em: 2 de março 2020.

OpenVibe (2019). Software para interface cérebro computador (BCI) e neurociências de tempo real. http://openvibe.inria.fr/discover/. Acesso em: 2 de março 2020.

Renard, Y, Lotte, F., Gibert, G., Congedo, M., Bertrand, O., Delannoy, V., Lécuyer, A., \& Maby, E.. (2010). OpenViBE: An Open Source Software Platform to Design, Test and Use Brain-Computer Interfaces in Real and Virtual Environments. Presence: Teleoperators and Virtual Environments. Massachusetts Institute of Technology Press (MITPress), p.35-53.

Saeid Sanei e J.A. Chambers. (2007). EEG SIGNAL PROCESSING. Centre of Digital Signal Processing Cardiff University. https://www.academia.edu/35159329/EEG_Signal_Processing. Acesso em: 2 de março 2020.

Toda Matéria (2020). Anatomia do Cérebro Humano. https://www.todamateria.com.br/cerebro/. Acesso em: 2 de março 2020.

WHO (2013). Organização Mundial da Saúde. https://www.who.int/news-room/ fact-sheets/detail/spinal-cord-injury. Acesso em: 2 de março 2020.

Wolpaw, J. R., 1, Birbaumer, N., Heetderks, W. J., McFarland, D. J., Peckham, P. H., Schalk, G., Donchin, E., Quatrano, L. A., Robinson, C. J., \& Vaughan, T. M., (2000) Brain-computer Interface Technology: A Review of the First International Meeting. EEE transactions on rehabilitation engineering : a publication of the IEEE Engineering in Medicine and Biology Society. 